\title{
Panorama atual e perspectivas futuras da cadeia produtiva de flores tropicais: o caso do antúrio(1)
}

\author{
LILIAN CRISTINA ANEFALOS(2), ANTONIO FERNANDO CAETANO TOMBOLATO(2,3) e ALBERTO RICORDI(4)
}

\begin{abstract}
RESUMO
O objetivo deste artigo é ampliar o conhecimento sobre as flores tropicais, com relação a aspectos relevantes de sua produção e comercialização, para que ações mais efetivas possam ser tomadas facilitando a ampliação da importância socioeconômica desse setor no Brasil, a partir da elevação do desempenho da cadeia e ampliação da participação brasileira no exterior. A pesquisa foi realizada em 2008, durante importante feira, na cidade de Holambra, SP, mediante entrevistas pessoais, a partir de questionário previamente estruturado. Os dados resultantes da pesquisa foram analisados, inicialmente, por meio de análise descritiva. Para que as relevantes informações fossem agregadas em grupos diferenciados, a fim de compor o panorama do setor de acordo com aspectos de produção e comercialização levantados neste estudo, foram utilizadas análises estatísticas complementares: análise fatorial de correspondência e de análise de conglomerados. Verificou-se que novas tecnologias foram absorvidas mais facilmente, como é o caso de opção por mudas microprapagadas, adoção de cultivos orgânicos, em função de maior disposição em buscar e incorporar novas técnicas, seja por meio de cursos ou de serviços de consultoria. De maneira geral, a pesquisa indica que há pouca informação sobre o segmento de flores tropicais. Apesar disso, nota-se que há interesse crescente por essas flores. Em relação ao antúrio, um dos principais produtos tropicais, constatouse que há perspectivas futuras positivas para elevação de sua produção, comercialização ou consumo, tanto no mercado interno quanto no externo. Para que possa haver crescimento das cadeias produtivas de flores tropicais, ações mais efetivas deverão ser tomadas para intensificar o acesso de novas tecnologias junto a cooperativas e produtores e para promover novos produtos no mercado interno, junto a atacadistas, varejistas e consumidores finais.
\end{abstract}

Palavras-chave: Anthurium andraeanum; cadeia produtiva; floricultura; antúrio.

\section{ABSTRACT}

Current panorama and future perspectives of the productive chain of tropical flowers: the case of the anthurium

\begin{abstract}
The objective of this article is to extend the knowledge on the tropical flowers, with regard to excellent aspects of its production and commercialization, so that actions more effective can be taken facilitating the magnifying of the socioeconômica importance of this sector in Brazil, from the rise of the performance of the chain and magnifying of the Brazilian participation in the exterior. The research was carried through in 2008, during important fair, in the city of Holambra, SP, by means of personal interviews, from questionnaire previously structuralized. The resultant data of the research had been analyzed, initially, by means of descriptive analysis. So that the excellent information were added in differentiated groups, in order to compose the panorama of the sector in accordance with aspects of raised production and commercialization in this study, had been used complementary statistical analyses: factorial analysis of correspondence and analysis of conglomerates. It was verified that new technologies had been absorbed more easily, as is the case of option for microprapagadas changes, adoption of organic cultivos, in function of bigger disposal in searching and incorporating new techniques, either by means of courses or of services of consultoria. In a generalized manner, the research indicates that it has little information on the segment of tropical flowers. Although this, notices that it has increasing interest for these flowers. In relation to the anthurium, one of the main tropical products, evidenced that it has positive future perspectives for rise of its production, commercialization or consumption, as much in the domestic market how much in the external one. So that it can have growth of the productive chains of tropical flowers, actions more effective will have to be taken to intensify the access of new technologies next to cooperatives and producers and to promote new products in the domestic market, next to wholesalers, final retailers and consumers.
\end{abstract}

Keywords: Anthurium andraeanum; productive chain; floriculture.

\section{INTRODUÇÃO}

No mercado mundial de flores e plantas ornamentais a concorrência entre os países exportadores tem_se elevado. Um dos fatores principais para essa situação é a oferta crescente de produtos de qualidade e com grande variedade para suprir a demanda de consumidores exigentes, em países com renda per capita mais elevada.
A floricultura empresarial brasileira vem adquirindo notável desenvolvimento e se caracteriza já como um dos mais promissores segmentos da horticultura intensiva no campo dos agronegócios nacionais (JUNQUEIRA e PEETZ, 2008). Levando-se em conta a elevada demanda de mão-de-obra por área quando comparada a agricultura extensiva, a floricultura contribui para a fixação de mão-de-obra no campo e torna-se adequada como cultura alternativa para pequenos produtores.

\footnotetext{
(1) Recebido para publicação em 27/08/2009 e aprovado em 04/03/2010.

(2) Instituto Agronômico, IAC, Avenida Barão de Itapura, 1481, CEP 13012-970 Campinas SP. E-mail: 1canefal@iac.sp.gov.br

(3) Bolsista $\mathrm{CNPq}$

(4) Master Student, University of Hawaii, USA.
} 
O Brasil apresenta potencial para tornar-se um grande produtor e exportador de flores e plantas ornamentais, destacando-se as tropicais, devido à grande diversidade genética e ao clima propício para sua produção. A área cultivada de flores e plantas ornamentais tem se expandido de forma contínua, de acordo com dados do IBRAFLOR (2008). Atualmente no Brasil registram-se mais de 6 mil hectares de área total, englobando 304 municípios e 12 pólos de produção e gerando mais de 120 mil empregos. Em relação à área total, cerca de $50 \%$ destinam-se à produção de mudas, $42 \%$ de flores (de corte e envasadas) e $8 \%$ de folhagens e demais produtos. Embora tenha havido expansão das áreas de produção de flores e plantas ornamentais para outros estados de todas as regiões do país, o estado de São Paulo ainda concentra a maior parte das áreas de cultivo e, também, a principal fonte de escoamento para a comercialização interna e externa. Outros pólos também se destacam, como é o caso do Rio Grande do Sul, Paraná, Santa Catarina, Minas Gerais, Rio de Janeiro, Goiás, Distrito Federal e na maioria dos estados do Norte e do Nordeste (IBRAFLOR, 2008).

As principais espécies tropicais cultivadas concentram-se na América tropical, Ásia e Pacífico Oeste (ASSIS et al, 2002). No Mercado de Flores da CEASA - Campinas, por exemplo, o maior mercado de plantas ornamentais do País, de acordo com VERA (2008), o comércio de flores tropicais ainda é incipiente. Há, portanto, potencial ainda não explorado, para que possa ocorrer expansão de sua oferta e comercialização no Brasil e exterior.

Como no setor de flores estão inseridos bens de maior valor agregado, é importante que se busquem novos nichos de mercado, com renda e consumo per capita mais elevados, e que ocorram investimentos em material de propagação, tecnologia e equipamentos, para que os produtos finais do setor de flores mantenham qualidade e se diferenciem dos demais.

Atualmente o antúrio constitui a principal espécie de flor tropical de interesse econômico no Brasil, cultivado principalmente no Vale do Ribeira, e neste processo de expansão dos negócios, o Instituto Agronômico (IAC) tem contribuído sobremaneira para o desenvolvimento de variedades adaptadas às condições climáticas e de cultivo do País, por meio de programa contínuo de melhoramento genético do antúrio. De acordo com TOMBOLATO et al. (2004), esses novos materiais genéticos estão sendo micropropagados em laboratórios especializados para cultivo comercial no Brasil.

Segundo NOMURA (2008), como a floricultura exige uso contínuo de tecnologias de ponta, tem se tornado setor altamente competitivo e dinâmico, principalmente com relação ao seu sistema de distribuição. Há necessidade de monitoramento do produto ao longo de toda a cadeia, para garantir escoamento da produção interna e a comercialização de flores e plantas ornamentais nos mercados doméstico e internacional, e, de acordo com ANEFALOS (2004), a infra-estrutura logística é um dos pontos vitais para que essas etapas sejam bem sucedidas. Entretanto, com relação às flores tropicais, segundo VERA (2008), não tem havido fornecimento adequado de informação aos consumidores sobre a qualidade e os diferenciais desses produtos, o que tem limitado o aumento e fluxo contínuo de seu consumo. Esse fato tem influenciado negativamente na elevação de sua demanda, já que o consumidor final não consegue obter parâmetros mais sólidos sobre esses produtos ofertados.

O objetivo dessa pesquisa é ampliar o conhecimento sobre as flores tropicais, com relação a aspectos relevantes de sua produção e comercialização, para que ações mais efetivas possam ser tomadas facilitando a ampliação da importância socioeconômica desse setor no Brasil, a partir da elevação do desempenho da cadeia produtiva e ampliação da participação brasileira no exterior.

\section{MATERIAL E MÉTODO}

Os dados primários foram coletados junto a produtores, profissionais e técnicos, durante importante feira brasileira, Hortitec, que congrega expositores dos setores de horticultura e fruticultura, relacionada a cultivo protegido e a culturas intensivas.

A pesquisa foi realizada mediante entrevistas pessoais, a partir de questionário previamente estruturado, durante o primeiro dia da feira, em 2008, realizada na cidade de Holambra, São Paulo. Os entrevistados foram abordados aleatoriamente em todo o espaço da feira, para que se pudesse garantir que os resultados representassem melhor o perfil de seus participantes. Apesar de o universo amostral ter sido mais restrito, em relação ao total de mais de 20 mil visitantes registrados na feira, de acordo com HORTITEC (2009), reflete disposição e interesse ao fornecimento de informações ao setor. Foram entrevistadas 31 pessoas durante a feira.

Para suprir as informações necessárias ao estudo, optouse por respostas binárias, que podiam ser justificadas pelos entrevistados durante a entrevista. O questionário foi dividido em duas partes:

- a primeira, com perguntas relacionadas ao perfil do entrevistado (faixa etária, sexo, ramo de atividade), sua experiência no setor de flores (conhecimento de flores tropicais, cultivo de alguma flor de vaso), e sua visão sobre a necessidade de investimento no setor de flores tropicais, tanto na produção (principais dificuldades com substratos, opção por cultivos orgânicos, problemas com a aplicação de agrotóxicos) como na comercialização desses produtos (uso de serviços de assessoria (consultoria) para cultivar uma nova espécie e/ou nova técnica de cultivo, disposição em pagar mais por flores cultivadas em sistema orgânico, em realizar cursos para aprimorar sua produção, em investir em mudas micropropragadas, pagar royalties para cultivo de uma variedade);

- a segunda concentrou-se em obter informações mais específicas à cadeia produtiva do antúrio (variedades de antúrio conhecidas, experiência com o cultivo de antúrio, cultivo mais atrativo para antúrio, relação de variedades de antúrio cultivadas, comercializadas ou compradas, período de tempo viável para produção de antúrio em vaso, principal mercado de venda de antúrio, outros mercados de venda de antúrio com potencial no futuro).

Os dados resultantes da pesquisa foram analisados, inicialmente, por meio de análise descritiva. Para que 
as relevantes informações fossem agregadas em grupos diferenciados, a fim de compor o panorama do setor de acordo com aspectos de produção e comercialização levantados neste estudo, foram utilizadas análises estatísticas complementares: análise fatorial de correspondência e de análise de conglomerados.

A partir da análise de correspondência é possível analisar dados qualitativos, agrupando-os e reduzindo o número de variáveis do modelo, para facilitar o entendimento das informações. Maiores detalhes sobre esses métodos poderão ser encontrados em HAIR et al. (1995), CRIVISQUI (1993), BENZÉCRI (1992), ESCOFIER e PAGÈS (1992) e GREENACRE (1984).

A análise de conglomerados ("cluster") permite que se construam grupos distintos, de acordo com medidas de similaridade. Essas divisões podem ser realizadas a partir dos resultados obtidos da análise de correspondência e permitem que se visualizem, de maneira mais clara, os fatores que agregam cada um dos grupos de indivíduos - neste caso - os entrevistados. DILLON e GOLDSTEIN (1984) descrevem mais detalhadamente os algoritmos utilizados neste método, para que se classifiquem os grupos.

\section{RESULTADOS E DISCUSSÃO}

Os entrevistados foram representados em sua maioria por homens, $65,5 \%$ do total. Os principais ramos de atividade são: produtores $(29 \%)$ e engenheiros agrônomos $(19 \%)$, conforme mostra a figura 1 .

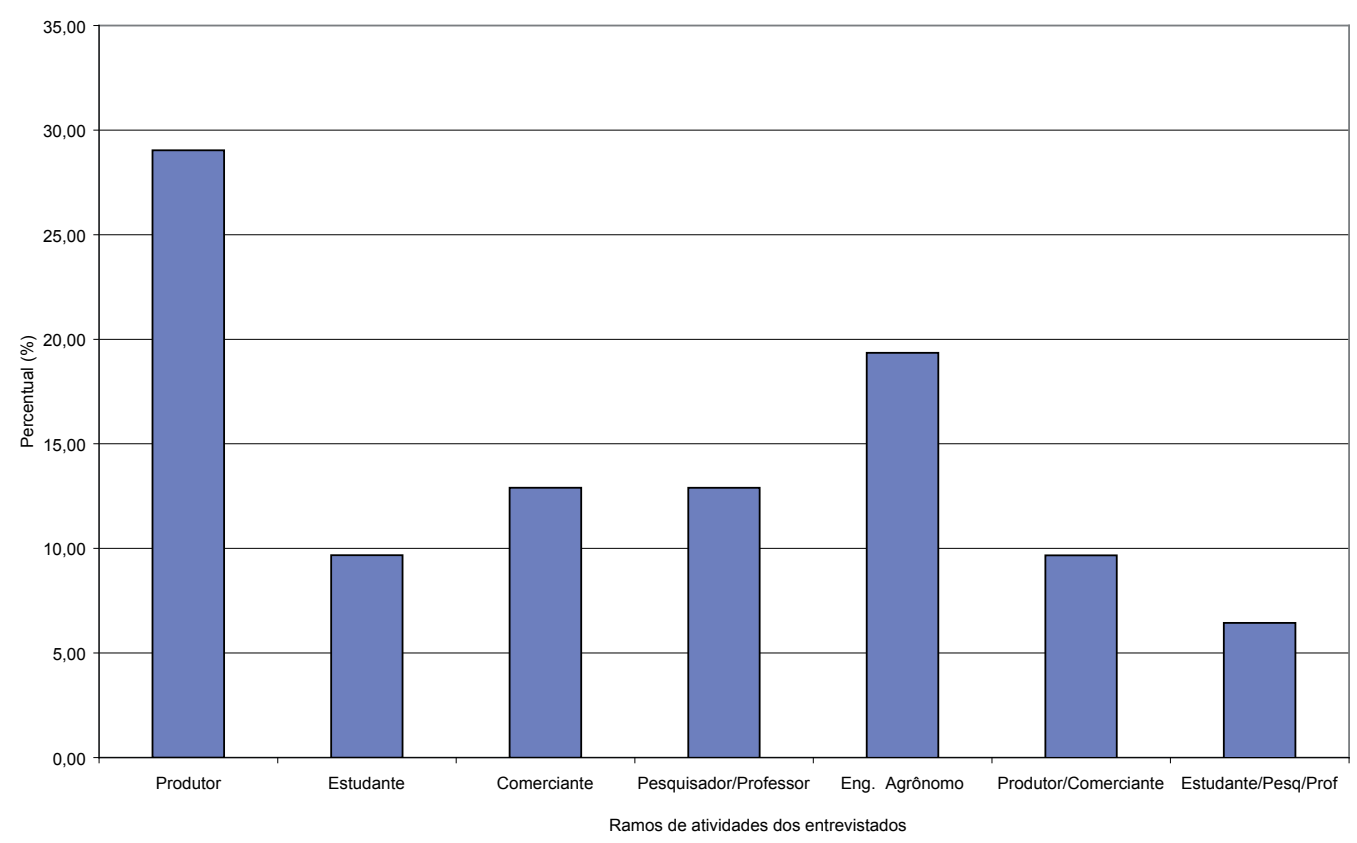

Figura 1. Ramos de atividades dos entrevistados

Em torno de $61,9 \%$ dos entrevistados informou conhecer flores tropicais e cerca de $58 \%$ já cultivaram flores em vaso.

A produção de flores exige aprimoramento permanente, tanto em técnicas quanto em introdução de novos materiais genéticos, que atendam a demanda do mercado. Dentre os produtores que foram entrevistados, cerca de 93\% já aperfeiçoa sua produção por meio de novos conhecimentos obtidos em cursos. Embora mais da metade dos entrevistados afirmou que não teve problemas com aplicação de agrotóxicos, $32 \%$ enfrentaram dificuldades ocasionadas por reações como fitotoxidez, infecção nos olhos do aplicador, alergias, e por resistência dos funcionários em utilizar equipamentos de EPI. Outro problema identificado diz respeito aos substratos $(61 \%$ tiveram dificuldades para sua utilização). Os principais motivos apontados foram sua qualidade, falta de informação sobre esse material e os preços elevados praticados no mercado.

Com relação à introdução de novas cultivares ou adoção de novas técnicas de cultivo, $71 \%$ consideraram relevante utilização de serviços de consultoria, para facilitar a aquisição de novas tecnologias e viabilizar a produção de produtos de melhor qualidade. A adoção do sistema orgânico ainda não é consenso entre os entrevistados: $29 \%$ já cultivam nesse sistema, $32 \%$ estarem dispostos a cultivar organicamente, porém $61 \%$ não adotam quaisquer sistema orgânico e cerca de $16 \%$ não pretendem adotar esse sistema. Dentre as justificativas para sua adoção destaca-se: representar produtos de maior valor agregado, com diferencial na comercialização, principalmente em se tratando de exportação, atrelando-os a produtos ambientalmente adequados, desde que tenham qualidade superior aos demais. A maior parte não se disporia a pagar mais por flores cultivadas em sistema orgânico.

Em relação às mudas micropropagadas, 97\% consideram que representam bom investimento no setor de flores, desde que haja garantia de qualidade do produto, com produção de clones melhorados, com maior homogeneidade e resistência a pragas e doenças. O pagamento de "royalties" para cultivar variedades também teve boa aceitação entre os entrevistados (71\%). Apesar de trazer benefícios para o melhorista, se os 
custos forem muito elevados, a adoção desses novos materiais pelos produtores poderá ser prejudicada.

A diversidade de materiais genéticos de flores temperadas e tropicais disponíveis no mercado é muito ampla. As flores tropicais ainda constituem-se em nicho de mercado mais restrito, por se tratar de espécies que estão em estado inicial de exploração comercial, com utilização menor de tecnologias avançadas no sistema produtivo e menor desenvolvimento de novas variedades. Dentre essas flores, destaca-se o antúrio, que tem tido boa aceitação no mercado interno e exterior. $\mathrm{Na}$ opinião de $42 \%$ dos entrevistados, o antúrio de corte mostra-se como cultivo mais atrativo e $23 \%$ consideram que ambos, corte e vaso, são boas opções para o antúrio. Um dos parâmetros relevantes para o cultivo de antúrio em vaso é o seu tempo de produção. Para $16 \%$ dos entrevistados o plantio em vaso deveria ser produzido em no máximo 6 meses, enquanto para $25 \%$ do total esse período poderia variar entre 1 a 3 anos.

Ao relacionar as principais variedades de antúrio, é interessante observar que a grande maioria dos entrevistados não estava familiarizada com seus nomes. Foram mencionadas: Cananéia, Choco, Eidibel, Fantasia, Iguape, Desiree, Juréia, Krenak, Leny, Midori, Netuno, Pistache, Rubi, Safári, Tropical e Verino. Em torno de $61 \%$ nunca cultivaram antúrio e 23\% já tiveram alguma experiência com plantio dessas flores.

Dentre os entrevistados que produziram antúrio, observouse que a maior parte cultivou essa flor nos últimos 5 anos e pequeno percentual optou pelo seu plantio entre 5 a 10 anos ou há mais de 10 anos atrás. Esse fato indica que tem havido interesse mais acentuado no cultivo dessa flor nos últimos 5 anos em relação a anos anteriores. Com relação à sua comercialização, o atacado e o varejo foram apontados como principais mercados de venda desse produto tropical, e o mercado externo representou opção potencial, a ser explorada no futuro.

Com o intuito de analisar mais profundamente os diversos tipos de informações obtidas das entrevistas, utilizou-se análise fatorial de correspondência para reduzir as 20 variáveis analisadas (perguntas), agregando-as em dois fatores.

A partir da análise de conglomerados ${ }^{(5)}, 4$ grupos distintos de entrevistados foram obtidos.

O Grupo 1 foi formado por dois entrevistados, de ramos distintos - produtor e comerciante. Possuíam alguma experiência em cultivo de flores e adotavam cultivo orgânico. Estavam dispostos a aprimorar conhecimentos no ramo por meio de cursos e eram favoráveis à introdução de novas técnicas por meio de serviços de consultoria. Nesse grupo todos conheciam alguma flor tropical ou já cultivaram alguma flor em vaso. Nenhum deles conhecia nomes de variedades de antúrio, nem cultivaram, comercializaram ou compraram antúrios, porém pretendiam comercializar essa flor no futuro.

No Grupo 2 os 11 entrevistados não estavam dispostos a adquirir novos conhecimentos, porém possuíam interesse em investir em mudas micropropagadas. Quanto às flores tropicais, apenas dois deles citaram alguns nomes, porém nenhum dos entrevistados identificou nomes de variedades de antúrio mais conhecidas no mercado. A maioria não teve experiência com cultivo de antúrio e nenhum deles cultivava, comercializava ou comprava flores tropicais. Apesar disso, a maioria considerava interessante cultivo de antúrio para corte, com potencial de comércio no mercado interno e exportação.

O Grupo 3 foi composto por produtores, em sua maioria. Todos os 12 entrevistados deste grupo tinham disposição em investir em mudas micropropagadas. Embora a maioria não tinha experiência no cultivo de antúrio, nominaram algumas flores tropicais e algumas variedades de antúrio presentes no mercado. A maior parte considerou atrativos os cultivos de antúrio para vaso ou corte, com foco atual no mercado interno e com potencial futuro no mercado doméstico e exterior. No caso de produção de antúrio de vaso, o período de 6 meses a 1 ano foi considerado o mais viável.

No Grupo 4 a maioria dos entrevistados eram professores e pesquisadores. Estavam dispostos a investir em mudas micropropagadas e consideraram importante o uso de serviços de assessoria. A maioria já cultivou alguma flor em vaso e estaria disposto a pagar mais por flores orgânicas. No entanto, os 6 entrevistados deste grupo não possuíam experiência no cultivo de antúrio e não tinham interesse em comercializar essa flor.

\section{CONSIDERAÇÕES FINAIS}

O mercado de flores tropicais ainda é muito incipiente no Brasil. É interessante observar que ao restringir o público alvo a pessoas com interesse em produtos hortícolas, ainda há pouca informação e experiência com relação a esse ramo da floricultura.

Por outro lado, nota-se que alguns conceitos importantes estão sendo incorporados mais facilmente, como é o caso de: opção por mudas microprapagadas; adoção de cultivos orgânicos; aderência maior à obtenção de novos conhecimentos para incorporar novas técnicas, seja por meio de cursos ou de serviços de consultoria.

De maneira geral, a pesquisa indica que entre os produtores hortícolas há pouco conhecimento difundido sobre o segmento de flores tropicais. Apesar disso, constata-se que há interesse nessa opção de negócio. Em relação ao antúrio, um dos principais produtos tropicais, há perspectivas futuras positivas para elevação de sua produção, comercialização ou consumo, tanto no mercado interno como no exterior.

Para que possa haver crescimento da cadeia de flores tropicais, ações mais efetivas deverão ser tomadas para intensificar o acesso de novas tecnologias junto a cooperativas e produtores e para promover esses novos produtos no mercado interno, junto a atacadistas, varejistas e consumidores finais. A ação das instituições de pesquisa na transferência de tecnologia é vital para que se consolide a difusão de novas variedades entre os produtores, por meio de ações direcionadas a eles, com maior divulgação de material informativo. Esse suporte tecnológico é fundamental para ampliação dos volumes de produção e renda,

${ }^{(5)}$ Utilizou-se, inicialmente, o método hierárquico Ward para obtenção do número ótimo de 4 grupos. A composição final de cada grupo foi obtida por meio do método não-hierárquico $k$-means. 
que viabilizem novos arranjos produtivos. Neste processo, é de especial importância também a ação de técnicos, consultores e empresários rurais, para disseminação de novos produtos tropicais e conquista dos consumidores, dispostos a adquirir essas flores com características diferenciadas no mercado.

\section{REFERÊNCIAS}

ANEFALOS, L. C. Modelo insumo-produto como instrumento de avaliação econômica da cadeia de suprimentos: o caso da exportação de flores de corte. Piracicaba: Escola Superior de Agricultura "Luiz de Queiroz", Universidade de São Paulo, 2004. 210p. Tese (Doutorado).

ASSIS, S.M.P., MARINHO R.R.L., GONDIM Jr., M.G.C., MENEZES, M. \& ROSA, R.C. T. Doenças e pragas de helicônias. Diseases and pests of heliconias. Recife: UFRPE. 2002.

BENZECRI, J. P. Correspondence Analysis Handbook, New-York : Marcel Dekker, P. 1992, 665p.

CRIVISQUI, E. M. Analisi Factorial de Correspondencias: un instrumento de investigación en ciencias sociales. Asunción: Universidad Católica "Nuestra Señora de la Asunción", 1993. $302 p$.

DILLON, W. R.; GOLDSTEIN, M. Multivariate analysis methods and applications. New York: Wiley, 1984. 587p.

ESCOFIER, B.; PAGÈS, J. Análisis factoriales simples y múltiples: objetivos, métodos e interpretación. Bilbao: Universidad del Pais Vasco, 1992. 285p.
HAIR, J. F.; ANDERSON, R. E.; TATHAM, R. L.; BLACK, W. C. Multivariate data analysis with readings. 4 ed. New Jersey: Prentice-Hall, 1995. 745p.

GREENACRE, M. J. Theory and applications of correspondence analysis. Orlando: Academic Press, 1984, $364 p$.

HORTITEC. 2009. Disponível em: http:// www.hortitec.com.br/comofoi.asp. Acesso em: 17 maio 2009 IBRAFLOR. Floricultura no Brasil: apontamentos mais relevantes sobre o papel sócio-econômico recente da atividade. Disponível em http://www.ibraflor.org/userfiles/ file/Floricultura $\% 20$ no\%20Brasil\%20- \%20atualizado $\% 2006$ .2008.pdf. Acesso em: 26 janeiro 2009

JUNQUEIRA, H.A.; PEETZ, M.S. Mercado interno para os produtos da floricultura brasileira: características, tendências e importância sócio-econômica recente. Revista Brasileira de Horticultura Ornamental, Campinas, v.14, n.1, p. 37-52, 2008.

NOMURA, E.S. Diagnóstico da produção de flores tropicais na região do Vale do Ribeira. Revista Brasileira de Horticultura Ornamental, Campinas, v.14, n.1, p. 23-25, 2008.

TOMBOLATO, A. F. C.; MATTHES, L.A.F. ; UZZO, R. P. ; CASTRO, A.C.R.; SAKAI, M.; SAES, L. A. Recursos genéticos e melhoramento do antúrio (Anthuirum andraeanum Linden) no IAC APTA. Revista Brasileira de Horticultura Ornamental, Campinas, v. 10, n. 1-2, p. 1-5, 2004.

VERA, R.M. Diagnóstico do mercado de flores tropicais. Revista Brasileira de Horticultura Ornamental, Campinas, v.14, n.1, p. 35-36, 2008. 